Canadian Journal of Regional Science

Revue canadienne des sciences régionales

\title{
Pour ou contre habiter une municipalité : discours sur les projets de communautés rurales au Nouveau-Brunswick
}

\section{Michelle Landry et Julie Guillemot}

Volume 44, numéro 1, 2021

Soumis : 13 mai 2019

Accepté : 1 octobre 2020

URI : https://id.erudit.org/iderudit/1079132ar

DOI : https://doi.org/10.7202/1079132ar

\section{Aller au sommaire du numéro}

\section{Éditeur(s)}

Canadian Regional Science Association / Association canadienne des sciences régionales

\section{ISSN}

0705-4580 (imprimé)

1925-2218 (numérique)

Découvrir la revue

Citer cet article

Landry, M. \& Guillemot, J. (2021). Pour ou contre habiter une municipalité : discours sur les projets de communautés rurales au Nouveau-Brunswick.

Canadian Journal of Regional Science / Revue canadienne des sciences régionales, 44(1), 11-21. https://doi.org/10.7202/1079132ar

\section{Résumé de l'article}

$\mathrm{Au}$ Canada, on associe aux municipalités deux principales fonctions, soit l'administration de services locaux et la gouvernance de la vie collective à l'échelle locale. Au Nouveau-Brunswick, où le tiers de la population habite à l'extérieur des municipalités, les débats et les mobilisations à propos de la municipalisation de territoires se multiplient depuis plus de 10 ans. Il s'agit donc d'un terrain privilégié pour étudier les fonctions attribuées aux municipalités. Nous présentons les résultats d'une analyse du contenu d'entrevues faites auprès d'acteurs impliqués dans de telles démarches. Le discours de ces acteurs, devant peser le pour et le contre de se doter d'un gouvernement local, révèlent la perception des rôles et les fonctions associées aux municipalités et l'importance relative qui y est attribuée, permettant ainsi de mieux comprendre pourquoi certains acteurs se mobilisent pour et d'autres contre la municipalisation de leur localité. 


\section{CANADIAN JOURNAL OF REGIONAL SCIENCE REVUE CANADIENNE DES SCIENCES REGIONALES}

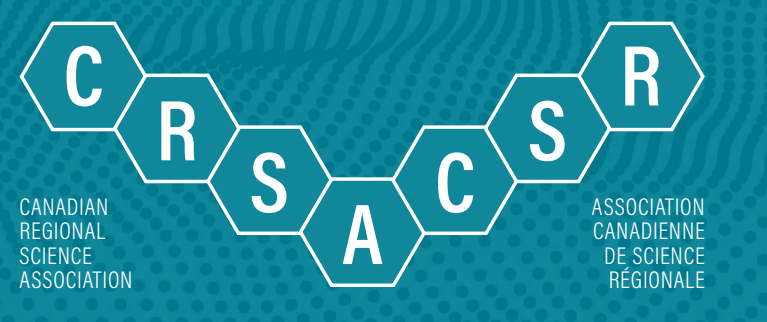

\section{POUR OU CONTRE HABITER UNE MUNICIPALITÉ: DISCOURS SUR LES PROJETS DE COMMUNAUTÉS RURALES AU NOUVEAU-BRUNSWICK}

\section{Michelle Landry, Julie Guillemot}

\section{Michelle Landry, Ph.D.}

Professeure agrégée de sociologie

Titulaire de la Chaire de recherche du Canada sur les minorités francophones canadiennes et le pouvoir Université de Moncton

Département de sociologie et de criminologie

(506) 858-4720

michelle.landry@umoncton.ca
Julie Guillemot, Ph.D.

Professeure (Bacc. Développement Durable et Zone Côtière) Université de Moncton, Campus de Shippagan

(506) 336-3400 poste: 365

julie.guillemot@umoncton.ca

Soumis: 13 mai 2019

Accepté : 1 octobre 2020

Résumé: Au Canada, on associe aux municipalités deux principales fonctions, soit l'administration de services locaux et la gouvernance de la vie collective à l'échelle locale. Au Nouveau-Brunswick, où le tiers de la population habite à l'extérieur des municipalités, les débats et les mobilisations à propos de la municipalisation de territoires se multiplient depuis plus de 10 ans. II s'agit donc d'un terrain privilégié pour étudier les fonctions attribuées aux municipalités. Nous présentons les résultats d'une analyse du contenu d'entrevues faites auprès d'acteurs impliqués dans de telles démarches. Le discours de ces acteurs, devant peser le pour et le contre de se doter d'un gouvernement local, révèlent la perception des rôles et les fonctions associées aux municipalités et l'importance relative qui y est attribuée, permettant ainsi de mieux comprendre pourquoi certains acteurs se mobilisent pour et d'autres contre la municipalisation de leur localité.

Mots Clés: Municipalisation, Canada, Nouveau-Brunswick, Gouvernement local, Districts de services locaux 
et les services de transport (nettoyage des rues et des routes muni-

Au Nouveau-Brunswick, $30 \%$ de la population habite des territoires non municipalisés (Statistique Canada, 2016'), c'est-à-dire sans gouvernement local, qui s'appelle districts de services locaux (DSL). Depuis l'adoption en 2005 d'une mesure volontaire visant à encourager les collectivités à se regrouper et à se municipaliser, des citoyens doivent évaluer les avantages et les inconvénients de se doter de telles institutions. Plusieurs localités de la province sont ainsi devenues le théâtre de vifs débats mettant en scène des citoyens qui se mobilisent en vue de se doter d'un gouvernement municipal et d'autres luttent pour maintenir le statu quo. Plusieurs chercheurs et analystes avant nous ont étudié des cas de fusions municipales, notamment en Ontario et au Québec. Ce texte se situe dans le même champ d'études, mais porte spécifiquement sur la municipalisation de territoires non incorporés, offrant par la nature même des débats, l'occasion unique de mieux comprendre les rôles attribués aux gouvernements locaux par les citoyens, notamment dans les petites localités où les services sont limités.

Notre objectif est de rendre compte de l'environnement discursif dans lequel ces projets de réformes en matière de gouvernance locale prennent part afin d'éclairer comment est perçu les rôles et fonctions principales des gouvernements locaux. II ne s'agit pas d'une étude sur le management municipal ni d'une étude comparative de cas. Nous n'avons pas non plus choisi l'angle de l'analyse d'une politique publique et de la construction des problèmes publics. Certains de ces thèmes pourront faire l'objet d'autres articles. Nous visons ici plutôt à contribuer aux connaissances sur le rôle perçu et l'importance relative attribués aux gouvernements locaux. Ces résultats pourront ainsi être pris en considération dans l'élaboration des politiques et des processus de restructuration municipale volontaires qui doivent tenir compte de la réalité sociale des résidents locaux, pour réaliser les réformes nécessaires.

\section{LE RÔLE ET L'IMPORTANCE DES MUNICIPALITÉS}

Les fusions municipales au Canada ont été amplement étudiées (par exemple: Sancton, 2000; Hollick \& Siegel, 2001; Nielson, Hsu \& Jacob, 2002; Sancton, 2003; Kushner \& Siegel, 2003; Rosenfeld \& Reese, 2003; McKay, 2004; Garcea \& LeSage, 2005; Douglas, 2005; Kushner \& Siegel, 2005; Lemire et coll., 2005; Hall \& Stern, 2009; Spicer, 2012). Les résistances aux fusions ont principalement été attribuées aux doutes quant aux économies annoncées, à la crainte de voir les impôts fonciers augmenter ou que certains quartiers se retrouvent à payer les services des autres (Kushner \& Siegel, 2003), ainsi qu'à une forte identité et un fort sentiment d'appartenance local (Hall \& Stern, 2009). Dans ces cas, le gouvernement local n'est pas remis en question, il est entendu que ce palier a sa pertinence et son utilité.

Le rôle effectif des municipalités et autres types de gouvernements locaux au Canada est aussi bien documenté dans la littérature (par exemple: Sancton, 2015; Tindal et al., 2013; Sancton \& Young, 2009; Antoft \& Novack, 1998). Au Canada, les municipalités ont été créées dans une logique de livraison de services de proximité et de gestion du territoire local (Collin \& Léveillée, 2003; Chiasson et al., 2014; Chiasson \& Mévellec, 2014; Andrew, 1999). Ce développement historique expliquerait ainsi pourquoi les municipalités sont principalement perçues et décrites comme des pourvoyeuses et gestionnaires de services (Chiasson et al., 2014; Finn 2008). Les principales responsabilités des municipalités sont en effet de procurer les services «durs» (entretien des routes, gestion des déchets, gestion de l'eau, etc.), les services locaux en matière de sécurité (police et pompier) cipales, signalisation, épandage de sel ou de sable en hiver, déblaiement, gestion du stationnement). Cependant, au cours des dernières décennies, les municipalités ont été amenées à porter des dossiers de plus en plus diversifiés. Les changements économiques et sociaux, étroitement liés au phénomène de la mondialisation, transforment le rôle et les fonctions des gouvernements locaux, notamment le contexte dans lequel ils opèrent (Andrew \& Goldsmith, 1998). Leurs responsabilités changent aussi au gré des relations intergouvernementales (McAllister, 2004). Les gouvernements municipaux sont en fait devenus incontournables dans le développement des territoires (Cleave \& Arku 2015; Courlet \& Pecqueur, 2013; Douglas, 2005). Les municipalités s'impliquent de plus en plus dans le développement économique et communautaire, par exemple dans les dossiers de création d'entreprises et d'emplois, de mise en place de logements abordables, d'aide à l'installation des immigrants et de développement du tourisme (Antoft \& Novack, 1998) ou à travers l'élaboration de politiques environnementales ou de stratégies d'adaptation au changement climatique (Guillemot, 2017). De plus, la plupart des municipalités s'occupent d'activités culturelles et de loisir comme l'organisation d'événements et la gestion de sites patrimoniaux, de musées et de centres récréotouristiques. Elles peuvent aussi jouer un rôle important en ce qui concerne l'aménagement et le paysage linguistiques (Foucher, 2012; Bourgeois, 2009; Landry, 2007).

Ces quelques exemples illustrent le fait que les rôles des municipalités, selon leurs moyens, vont bien au-delà de la gestion des services durs afin de prendre en charge des enjeux de différentes natures vécus sur leur territoire. La municipalité est par ailleurs souvent un acteur central dans la définition d'orientations collectives et la mise en œuvre de projets, dépassant souvent ses propres frontières (Mévellec et al., 2017). Cela implique d'une part que la définition de ces orientations collectives repose sur des bases démocratiques solides et d'autre part une redéfinition des relations du palier local avec les autres ordres de gouvernement (Young, 2013). En ce qui concerne le premier aspect, si les municipalités peuvent renforcer ces bases démocratiques dans le cadre de la définition de projets territoriaux, plusieurs observateurs du monde municipal canadien soulignent encore la "prédominance d'une gestion par la technocratie et l'expertise plus que par le débat idéologique et démocratique sur des projets de société, » (Chiasson et al., 2014: 68). Reste que les citoyens s'attendent tout de même à ce que le conseil municipal élu les représente et reflète l'expression de la volonté de la communauté locale (Tindal et al 2013: 9). Les municipalités sont ainsi aussi des institutions politiques locales (Mévellec et al., 2017; Tindal et al., 2013; Cyr, 2001). Comme Tindal et ses collaborateurs (2013) le résument:

On one hand, municipal governments are created by provinces and territories to deliver a wide range of necessary services, programs, facilities, and regulations to local residents. On the other hand, local residents expect elected municipal governments to express the will of the local community - even if what the community wants falls outside the authority of the municipal government to deliver or directly contradicts senior government policy (Tindal et al., 2013, p. 9).

Les municipalités ont donc deux principaux rôles: elles s'occupent de fournir et de gérer des services, des programmes, de l'infrastructure et de réglementer à une échelle locale; et elles procurent des mécanismes permettant aux citoyens de s'exprimer, de débattre et de résoudre des enjeux et des problèmes locaux (Tindal et al., 2013).

1 Nos calculs selon le statut des subdivisions de recensement (SDR), 
Ces constats, qui sont de l'ordre des évidences dans d'autres contextes, mettent en exergue l'exceptionnalité de la situation du Nouveau-Brunswick qui n'a jamais procédé à la pleine municipalisation de son territoire habité et où un important nombre de citoyens s'opposent aux projets visant à municipaliser leur localité. Dans la prochaine section, nous présenterons le développement historique de cet enjeu qui a abouti à la plus récente intervention, soit le règlement de 2005 permettant la création de municipalités ayant le statut de «communauté rurale», ainsi que d'autres changements dans la gouvernance locale qui ont suivi. Ce contexte est important pour comprendre quels rôles les acteurs attribuent aux gouvernements locaux et quelle importance ils leur accordent à travers le discours.

\section{DES EFFETS PERVERS D'UNE VACHE SACRÉE À LA "DÉPENDANCE DU SENTIER"}

Au Nouveau-Brunswick, dans les années 1960, une importante réforme fiscale a eu comme conséquence une réorganisation de la gouvernance locale. Le programme Chances égales pour tous est davantage connu pour avoir centralisé au niveau de la province les «services à la personne» : l'éducation, la santé, les services sociaux et l'administration de la justice, et par conséquent pour avoir considérablement réduit les disparités rurales-urbaines en matière de qualité de services et de taux d'imposition (Antoft \& Novack, 1998; Young 2001; Landry, 2007; Bourgeois \& Strain, 2009; Tindal et al., 2013). Cette réforme était nécessaire afin de faciliter la gestion des subventions fédérales pour le développement régional (Young, 2001).

Peu se souviennent ou accordent une importance au fait que les conseils de comté ont été abolis, laissant une grande partie des populations rurales sans gouvernance locale. Environ 90 villages ont été créés (Cyr, 2001), mais une importante partie de la population se situait toujours à l'extérieur de territoires municipalisés. La province a donc créé 250 districts de services locaux (DSL), des unités administratives vouées à la gestion des services dans le territoire résiduel. Se fiant aux théories de modernisation et d'urbanisation, le gouvernement prévoyait à l'époque que la population des DSL diminuerait (Martin, 2007). L'absence de démocratie locale n'était donc pas perçue comme un problème, d'autant plus que la province assurait les services dans les DSL et qu'à cette époque, c'était ce qui est important. Cette réforme est aussi à la source du régime d'imposition foncier en vigueur au Nouveau-Brunswick où le ministère des Finances et du Conseil du Trésor facture et perçoit les taxes foncières, y compris les impôts prélevés par les gouvernements locaux. Le taux provincial est uniforme sur le territoire pour les différentes catégories de biens et le taux municipal est déterminé par chaque gouvernement local pour son territoire. Pour ce qui est des districts de services locaux, c'est le ministre de l'Environnement et des Gouvernements locaux qui fixe le taux.

La coexistence de zones municipalisées et non municipalisées a cependant rapidement été dénoncée dès les années 1970 et les études sur la gouvernance locale et les réformes ont commencé à se succéder (Finn, 2008). Le gouvernement conservateur de Richard Hatfield (1970-1987) créa 12 commissions d'aménagement, 13 commissions de développement économique et 12 commissions de gestion des déchets. Le gouvernement libéral subséquent de Frank McKenna (1987-1997) procéda à quelques fusions municipales et à une réorganisation des commissions. Par exemple, les commissions scolaires ont été réduites à 2 , une de langue anglaise et une de langue française, on a réduit le nombre de régis de la santé et on a créé une seule commission de services régionaux pour les municipalités voisines de Moncton, Dieppe et Riverview. La question de la population non municipalisée n'a toutefois pas été abordée, probablement jugée trop risquée politiquement. À la suite de la démission de McKenna, le gouvernement libéral, sous la gouverne de Camille Thériault, a formé un panel sur la révision de la Loi sur les municipalités. Les recommandations de ce panel ont été présentées au gouvernement conservateur de Bernard Lord (1999-2006) qui a entre-temps pris le pouvoir. C'est ce gouvernement qui adopta le règlement sur la constitution et la restructuration d'une communauté rurale en vertu de la Loi sur les Municipalités. II s'agit de la première véritable tentative de proposer une solution au problème de la population non municipalisée (Finn, 2008).

La démarche de municipalisation est toutefois volontaire. Le règlement permet aux DSL de se regrouper, en intégrant parfois une ou des municipalités, afin de créer une nouvelle entité nommée «communauté rurale». Une communauté rurale peut acquérir progressivement les mêmes pouvoirs que les autres municipalités, mais elle a l'avantage de ne pas être responsable de l'entretien des routes sur le territoire des anciens DSL. Outre la création du statut de communauté rurale, la municipalisation du territoire peut se faire par l'annexion des DSL à une municipalité existante. Le discours analysé dans cet article découle ainsi des projets proposés sous l'égide de cette mesure volontaire.

Quelques réformes et études ont suivi la mise en place de cette nouvelle mesure, mais sans attaquer de front la municipalisation du territoire, même si plusieurs le demandent. En 2006, voulant améliorer la situation économique de la province, le gouvernement libéral de Shawn Graham (2006-2010) a mis sur pied le Groupe de travail sur l'autosuffisance du Nouveau-Brunswick. Dans son rapport, les commissaires recommandaient de créer «une commission sur l'avenir des gouvernements locaux en vue d'une réforme de l'organisation territoriale, structurale, législative et financière du système de gouvernance locale du Nouveau-Brunswick» (GNB, 2007). On nomma Jean-Guy Finn, un ancien haut fonctionnaire provincial, à la tête de cette commission qui déposa son Rapport sur l'avenir de la gouvernance locale, mieux connu comme le rapport Finn, en 2008. Ce rapport comprenait 97 recommandations portant sur les gouvernements locaux, les structures régionales et la livraison des services municipaux. La première recommandation est que «des gouvernements municipaux soient constitués sur l'ensemble du territoire du Nouveau-Brunswick et que tous les résidants [sic] soient représentés et gouvernés par des conseils municipaux élus, sauf en ce qui touche les Autochtones, lesquels ont une forme de gouvernement local distincte instituée en vertu d'une loi fédérale » (Finn, 2008, p. 199).

Le gouvernement libéral de Graham n'a toutefois pas donné suite au rapport Finn. Les conservateurs de David Alward (2010-2014) ont lancé un Plan d'action pour un nouveau système de gouvernance locale au Nouveau-Brunswick en 2011 qui a principalement abouti à la création de 12 commissions de services régionaux qui gèrent l'aménagement du territoire et la gestion des déchets, autrefois gérés par des commissions distinctes. Elles peuvent également faciliter la planification et le partage des coûts des plans d'urgence et des principales installations sportives, récréatives et culturelles de la région.

Enfin en 2018, une modernisation de la loi sur les municipalités et de la loi sur l'urbanisme très attendue a eu lieu sous le gouvernement libéral de Brian Gallant (2014-2018). Celles-ci donnent plus de pouvoir et d'autonomie aux gouvernements locaux, mais n'abordent pas la question du territoire et des citoyens qui n'en ont pas.

En fin de compte, pratiquement tous les gouvernements, qu'ils soient libéraux et conservateurs ont effectué des réformes en matière de gouvernance locale depuis les années 1960. Aucun n'a cependant abordé de front la question des territoires non municipalisés. Les raisons pouvant expliquer ce constat ne sont pas évidentes, il nous semble qu'il s'agit d'une forme de dépendance du sentier. Une réforme forcée aurait été et serait encore politiquement risquée. II n'a jamais eu de véritable appétit dans les DSL pour les réformes de gouvernance locale. Comme le montreront nos résultats d'entretien, 
les habitants de ces localités apprécient les taux d'imposition fonciers qui sont généralement moins élevés que dans les municipalités (Young, 2001) et craignent le changement pour des raisons qui seront expliquées plus bas. Un gouvernement qui imposerait une réforme en profondeur risquerait ainsi de devenir fort impopulaire, surtout dans les milieux ruraux où se trouve la majorité de la population sans gouvernement local. Ce système coûte cependant maintenant cher à la province et aux municipalités avoisinantes. Bourgeois \& Strain (2009) estimaient que la différence entre les impôts fonciers collectés dans les DSL et le coût réel des services offerts étaient de 39,6 millions de dollars en 2009. Ce type d'enjeux fiscaux sont une source de tension entre municipalités et communautés non incorporées depuis plusieurs années, d'autant plus que les populations périurbaines utilisent les services des municipalités avoisinantes (Martin 2007).

En somme, depuis 2005, cet enjeu est clairement relayé aux acteurs locaux qui souhaiteraient bien changer la situation dans leur localité. Ceux-ci doivent ainsi porter le projet à bout de bras, bénévolement, et essayer de convaincre la population locale des avantages, non seulement à se municipaliser, mais souvent aux fusions qui viennent avec. Comme nous le verrons, les discours qui ressortent de ces débats font grandement ressortir le rôle perçu et l'importance relative attribués aux gouvernements locaux. Ils soulignent ainsi à quel point, pour beaucoup de citoyens, les gouvernements locaux servent principalement à la livraison de services et à la règlementation locale.

\section{MÉTHODOLOGIE}

Les débats entourant les projets de création de communautés rurales au Nouveau-Brunswick offrent une occasion inédite pour comprendre l'importance et les rôles attribués aux gouvernements locaux. En effet, on ne discute pas de gouvernance municipale quotidiennement en temps normal, mais avec la proposition de projets locaux de création de communauté rurale dans différentes localités sans gouvernement local au Nouveau-Brunswick, nous avons eu accès à un matériel discursif riche et dense.

Nous avons ainsi opté comme collecte de données pour la conduite d'entrevues semi-directives avec des acteurs s'étant mobilisés pour ou contre les projets de communauté rurale dans leur localité. Afin d'assurer une cohérence dans le corpus et une meilleure compréhension, nous avons sélectionné 4 projets desquels extraire l'échantillon d'entretiens. Sans vouloir, ni pouvoir, faire une analyse comparative de cas, cette concentration des entretiens nous permet de mieux tenir compte d'enjeux locaux particuliers dans l'analyse et d'ainsi mieux comprendre le sens du discours des acteurs. Nous avons ensuite procédé à l'analyse thématique de contenu de ces entretiens afin d'en arriver à jeter un éclairage sur l'importance accordée aux gouvernements locaux.

\section{Sélection des projets faisant partie de l'échantillon}

À ce jour, 26 projets de création de communauté rurale sont passés à travers tout le processus prévu pour la municipalisation de DSL jusqu'au rejet ou l'acceptation du projet par plébiscite local ${ }^{2}$ (voir tableau 1).

Les projets visés pour l'échantillonnage d'entretiens ont été sélectionnés à la suite d'une l'analyse de la documentation (revue de presse et études de faisabilité) traitant des 17 démarches de création de communautés rurales ou municipalité régionale ${ }^{3}$. Bien qu'ils contribuent à la municipalisation du territoire, nous avons choisi
Tableau 1. Processus de municipalisation de DSL depuis 2005

\begin{tabular}{lrrr} 
& Échec & Succès & Total \\
\hline Création d'une communauté rurale & 9 & 8 & $17^{*}$ \\
Création d'une Municipalité régionale & 0 & 1 & 1 \\
Annexion à une municipalité & 3 & 4 & 7 \\
DSL devient une municipalité & 1 & 0 & 1 \\
TOTAL & 13 & 13 & $\mathbf{2 6}$ \\
\hline
\end{tabular}

*Quelques projets, parfois modifiés, ont fait l'objet de deux plébiscites avant d'être acceptés par la population (projets du Haut-Madawaska ou de Cocagne)

Sources : Élection N.-B. et Acadie Nouvelle (2005-2019

de ne pas couvrir les cas d'annexion de DSL à une municipalité (7) ou de transformation d'un DSL en une municipalité (1) pour nous concentrer sur le processus de création de communautés rurales afin de traiter du même type de projet. Aussi, certains de ces projets remontaient à trop longtemps pour y mener des entretiens, car nous aurions eu un trop grand effet de distorsion de la mémoire. Nous les avons donc exclus d'emblée. Par ailleurs, puisqu'au NouveauBrunswick le tiers de la population est francophone et que bien des enjeux peuvent être vécus et perçus différemment selon la communauté linguistique et culturelle d'appartenance, nous voulions un nombre égal de projets concernant des localités francophones et anglophones. II était aussi important qu'un nombre égal de projets acceptés et rejetés soit considéré pour l'échantillonnage. En plus des critères de la langue et du succès/échec des démarches, nous avons sélectionné les projets en tenant compte de la taille de la population visée par le projet, la présence ou non de municipalités dans le projet et la proximité d'un centre urbain, afin de tenter de couvrir une diversité de situations représentative du processus de création de communautés rurales au N.-B.

Finalement, les ressources et le temps alloués au projet de recherche permettaient d'effectuer des entrevues dans quatre régions. Deux (2) des cas sélectionnés sont donc des collectivités francophones et deux (2) sont anglophones et pour chaque communauté linguistique, une démarche sur deux a abouti à un rejet du projet. Les principales caractéristiques des quatre projets de municipalisation desquels sont tirés les entretiens de recherche sont présentées dans le tableau 2.

\section{L'entretien semi-dirigé de recherche}

L'entretien semi-dirigé a été retenu comme technique de collecte de donnée puisqu'il permet de rendre compte de la subjectivité des divers acteurs, tout comme il permet d'obtenir de l'information d'arrière-scène qui ne figure pas dans les documents (Savoie-Zajc, 2008). "L'exploration en profondeur de la perspective de l'acteur», nous apparaissait ainsi comme le principal argument épistémologique justifiant cette technique de collecte de donnée (Poupart, 1997) et nécessaire pour arriver à mieux comprendre les sens que donnent les acteurs à la possible municipalisation de leur localité, le rôle qu'ils attribuent aux municipalités, ainsi qu'au lien entre ces éléments.

Nous avons fait un échantillonnage de type non aléatoire, au jugé (Beaud, 2009) au sein des localités concernées pour les 4 projets retenus. En d'autres mots, une fois les projets ciblés sélectionnés, les participants ont été identifiés et recrutés par les chercheures et les assistants de recherche à l'aide d'informateurs clés. Un nombre approximatif d'entrevues avait été prévu selon le budget et nous avons

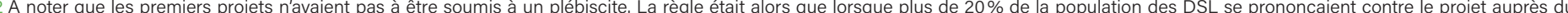

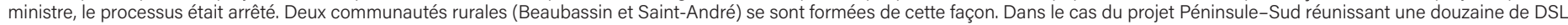
du secteur de Tracadie en 2006, le projet a été rejeté selon ce protocole.

3 La dix-huitième démarche inclue dans le tableau 1 a été soumis au plébiscite après la conduite des entretiens, le 6 mai dernier.
} 
Tableau 2. Présentation des quatre projets desquels est tiré l'échantillon

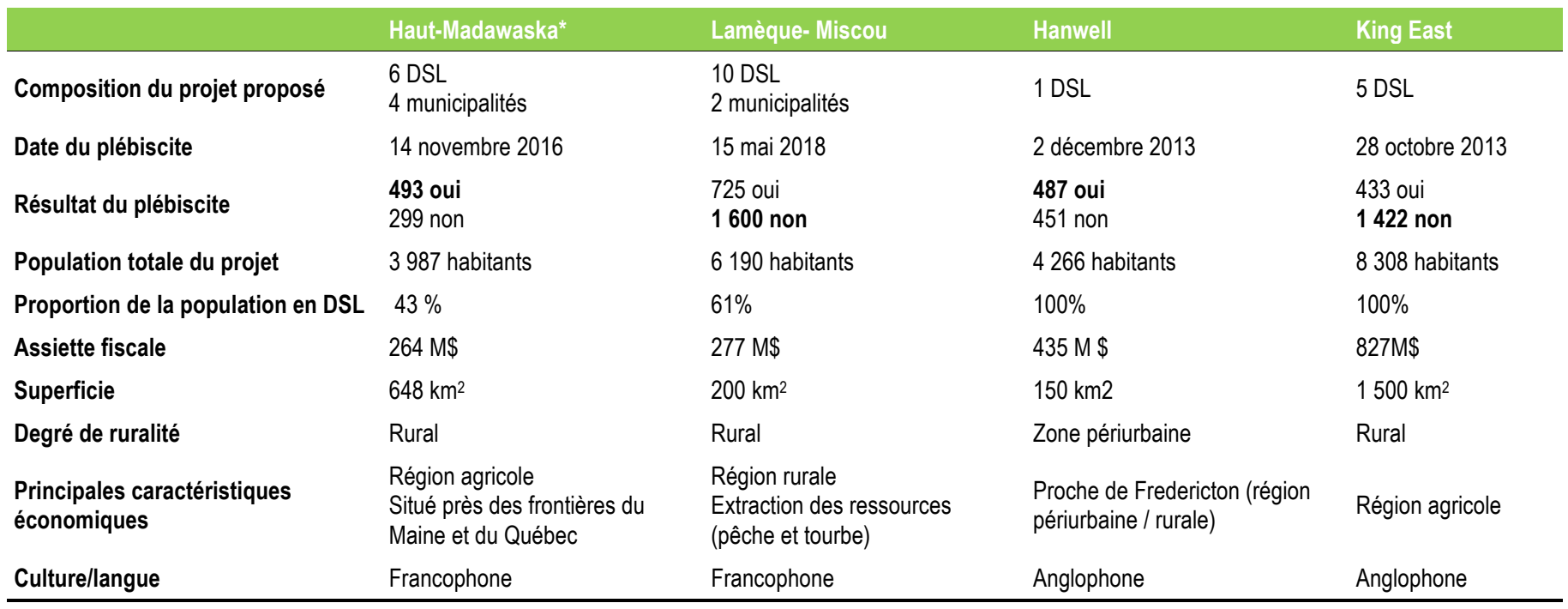

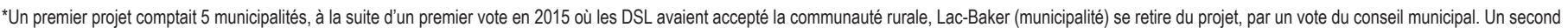
referendum est organisé en 2016 comprenant les mêmes collectivités sauf le village de Lac-Baker.

Sources: Élection N.-B. et rapports des études de faisabilité des 4 cas

largement atteint le principe de saturation (Savoie-Zajc, 2008). C'està-dire que les dernières entrevues n'ont pas apporté d'éléments d'une grande nouveauté nous permettant de mieux comprendre notre objet. Mis à part quelques enjeux fortement localisés, le discours des acteurs favorables ou défavorables ne diffère pas non plus grandement entre les localités.

Notre échantillon comprend 33 entrevues semi-dirigées avec des acteurs qui se sont mobilisés en faveur ou contre le projet proposé localement. Quatorze des participants étaient opposés au projet de création de communauté rurale proposé dans leur localité et 19 y étaient favorables. Nous avons aussi conduit 3 entretiens avec des acteurs gouvernementaux. Ce petit nombre s'explique par la petitesse de la fonction publique néo-brunswickoise et par conséquent le faible nombre de fonctionnaires impliqués dans ces démarches.

Le schéma d'entretien comprenait quelques questions visant à mieux saisir l'engagement communautaire du participant et à recueillir de l'information sur le projet et les localités ciblées. Ensuite, l'entretien portait principalement sur son implication dans le projet de création de communauté rurale ou dans le mouvement d'opposition, son opinion sur ce que sa communauté avait à gagner ou à perdre avec la restructuration. Nous avons aussi posé directement la question à savoir, à leur avis, quelles sont les différences entre une municipalité et un DSL et à quoi sert une municipalité.

\section{L'analyse}

Pour le volet du projet de recherche rendu compte par cet article, nous avons centré l'analyse sur les enjeux des débats au sujet des projets de création de communauté rurale et sur le discours entourant les rôles et fonctions des municipalités. II s'agit d'une analyse de contenu d'entretiens menés auprès d'acteurs visant à dégager les éléments constitutifs de la représentation (Negura, 2006) des gouvernements locaux. Les entretiens gouvernementaux ont seulement servi à titre informatif. L'analyse en recherche qualitative est généralement proche des données brutes et des contextes, le chercheur doit ainsi organiser cette information en un portrait cohérent qui dépasse la simple description pour atteindre ses objectifs (Neuman, 2010). C'est pourquoi nous avons codé et analysé les verbatims de ces entrevues à l'aide d'un logiciel d'analyse qualitative (MaxQDA) afin de faire émerger les thèmes et mieux comprendre leur articu- lation. Puisqu'il ne s'agit pas d'une analyse comparative de cas, les entretiens ont été analysés en lot selon la position des acteurs: pour ou contre.

Les résultats présentés dans cet article reposent ainsi sur une comparaison des discours des acteurs opposés au projet et du discours de ceux qui y étaient favorables, en se concentrant sur les catégories de codes qui relataient les enjeux et qui traitaient de gouvernance locale. De manière plus large, nous avons porté plus d'attention au matériel qui nous permet de mieux saisir la polarité entre la municipalité comme institution politique et comme organisation de prestation de service.

\section{DISCUSSION DES RÉSULTATS: POURQUOI VOUDRIONS-NOUS UN GOUVERNEMENT LOCAL?}

Comme nous le disions plus haut, l'enjeu de la municipalisation de localités sans gouvernement local s'apparente à plusieurs égards aux questions de fusions municipales, mais les débats qui se forment autour des projets de création de communautés rurales offrent une occasion quasi unique de mieux comprendre, au-delà des rôles effectifs des municipalités déjà bien documentés, ce que représente une municipalité pour les citoyens de petites localités où les services sont limités, à l'extérieur comme à l'intérieur de la municipalité. Nous arrivons ainsi à mieux comprendre ces questions en analysant le contenu du discours obtenu par entretiens de recherche au sujet des propositions de projets de création de communauté rurale. Rappelons que ce statut de municipalité permet de laisser certaines compétences, comme l'entretien des routes dans les anciens DSL, au palier provincial.

II importe également de souligner que 3 des 4 projets desquels ont été recruté les participants comprenaient un regroupement de DSL et de petites municipalités déjà existantes et que dans ces cas, les projets prévoyaient des taux d'imposition foncière différents d'une localité à l'autre au sein d'une même communauté rurale selon les services offerts. Le projet de Hanwell, quant à lui, ne consistait qu'à municipaliser sous le statut de communauté rurale un seul DSL. L'aspect de regroupement de localités compris dans les projets est souvent l'une des principales sources d'opposition aux projets, mais 
il s'agit aussi, pour d'autres, d'un avantage. Les résultats présentés ci-dessous seront cependant principalement organisés autour des deux grandes catégories de rôles associés aux gouvernements locaux dans la littérature, comme présenté plus haut, soit la prestation de services de proximité et leurs rôles politique et démocratique de représentation de la volonté et des intérêts de la collectivité.

\section{La municipalité comme prestataire de services}

Dans l'histoire canadienne, les municipalités ont principalement été créées pour livrer des services de proximité et de gérer le territoire local (Collin \& Léveillée, 2003; Chiasson et al., 2014; Chiasson \& Mévellec, 2014; Andrew, 1999). Comme le palier provincial a pris en charge les services à la population comme l'éducation et les services sociaux qui sont donc financés à partir de l'impôt provincial et les transferts fédéréaux, les municipalités sont restées avec les services «durs», comme l'entretien des routes, l'eau et les égouts, l'enlèvement des ordures et de la neige, etc., mais aussi les services associés à la sécurité locale (pompier, mesures d'urgence, etc.).

Les entrevues de recherche, autant celles conduites auprès d'acteurs en faveur du projet de communauté rurale dans leur localité que ceux contre font transparaître la prédominance de cette représentation de la municipalité, c'est-à-dire vue comme une organisation dont la principale raison d'être est de procurer des services liés à l'infrastructure.

Dans le cadre des débats sur la municipalisation, parmi tous les segments codés comme des enjeux, les sous-codes associés aux services sont les plus nombreux. Par ailleurs, ceux-ci sont nombreux à être associés au sous-code d'enjeu financier; nous y reviendrons.

Soulignons d'abord que pour les acteurs en faveur de la municipalisation, le gouvernement municipal est un gouvernement de proximité qui peut efficacement livrer les services locaux. Comme le montre cet extrait, c'est là son importance: "des niaiseries comme des lumières de rues qui marchent pu bien, c'est qui tu penses qu'ils appellent, y'appellent le conseil municipal. Ça fait que c'est important, ça prend ça » (HM2).

Dit autrement, le conseil municipal sert de porte à laquelle cogner lorsqu'on a des préoccupations au sujet des routes ou d'autres infrastructures:

Bien, présentement en $D S L$, une autre préoccupation que moi j'ai présentement, c'est justement au niveau des routes, des infrastructures. On sait que ça coûte quand même assez cher au gouvernement et vu qu'on est une petite communauté, disons que souvent notre tour est assez loin pour la réfection des routes et tout ça. Donc je pense que si on était municipalisé, là j'ai une porte à aller cogner pour dire par exemple hé en avant de chez nous on a besoin de faire des réparations et tout ça. Présentement, c'est très difficile pour un citoyen dans un DSL de faire valoir certains points, par exemple, ou apporter certaines améliorations dans sa communauté (LM11).

La prédominance de l'association entre les gouvernements locaux et la prestation de services «durs» s'est manifestée assez clairement lorsque les participants exprimaient ce qui est important, d'un point de vue local, pour les citoyens. Un participant de Hanwell explique que les routes ont beaucoup d'importance puisqu'elles sont visibles et sont utilisées quotidiennement: But roads are always an issue. People will complain about roads all the time because this is what they see. They see it. They feel it. They won't complain about recreational services or garbage collecting because ok it's done but the roads, every day they use the road so if there's something in the road they will complain (H11). Un autre participant, cette fois du projet Kings East, abonde dans le même sens: I think the biggest concern is roads, they like to have safe accessible roads then the next thing would be fire service and garbage pick-up so the community stays clean (KE3).

Or, ces services de base, comme l'entretien des routes, le service d'incendie et l'enlèvement des ordures sont quand même offerts dans les DSL. Ils sont simplement gérés à un autre niveau, soit par un ministère provincial, soit par la commission de services régionaux. Pour la plupart des opposants aux projets de création de communauté rurale, la ruralité et l'organisation communautaire font en sorte qu'un gouvernement local n'est pas nécessaire. Ce résident de l'île de Lamèque explique qu'une municipalité n'est pas nécessaire pour se doter des services et de l'infrastructure souhaités dans sa localité: "On a le droit d'avoir un centre communautaire [...]. On a le droit d'avoir une caserne de pompiers, on a le droit d'avoir des jeux pour enfants, $y^{\prime}$ a plein de choses qu'on peut avoir sans avoir une mairie» (LM7). Souvent, les opposants ne pensent pas que la municipalisation de leur communauté ni un regroupement, leur apporterait quoi que ce soit. Ils revendiquent un mode de vie rural et acceptent de se déplacer pour leurs services, comme l'illustre cet extrait:

In the rural area it's a different ball game, you don't need recreation, we don't have the infrastructure to have these things out, we need to go to the municipalities to do our things there, do our shopping, do our business there. The only way we're doing recreation in the rural area is as individuals like snowmobiling, 4-wheeling, horse back riding, hunting which are all things which are prohibited in town (KE4).

La municipalisation pour cette personne viendrait ainsi avec des règlements qui limiteraient le mode de vie rural.

Les partisans des projets de communautés rurales semblent cependant avoir une vision plus étendue des services, correspondant plus aux nouvelles fonctions prises en charge par les municipalités depuis quelques dizaines d'années. Ceux-ci essaient de promouvoir l'idée qu'il y aurait davantage de services pour tous en se regroupant pour obtenir le statut de communauté rurale. Le regroupement des communautés conjugué à l'accès au statut de municipalité leur permettrait d'attirer des investisseurs, développer le tourisme ou d'autres industries, d'éviter des compétitions entre collectivités voisines, d'optimiser l'usage des ressources humaines et matérielles et donc d'améliorer l'offre de services aux citoyens. Sans en être certain, plusieurs pensent qu'ils augmentent leur chance d'améliorer leurs services en se groupant, comme nous l'explique un résidant d'une communauté rurale du Nord-Ouest: "Y'aurait peut-être plus de services en se regroupant [...] j'pense que ça joué un rôle positif dans la décision des gens [vote en faveur du projet dans ce cas]» (HM3).

Mais comme ailleurs, les infrastructures sont concentrées dans les plus grandes collectivités du projet, déjà municipalisées. Les acteurs qui s'opposent aux projets de création d'une communauté rurale craignent ainsi souvent une accentuation de cette centralisation comme l'illustre cette citation: "Mais c'est quoi cette maladie-là que vouloir regrouper regrouper regrouper. [...] C'est pour [...] enlever les services, toute centraliser les services » (LM5). Certains, comme ce résident du Nord-Est, y voient même une perte de services, sup- 
posant qu'il s'agit d'un exercice de rationalisation déguisée: «parce qu'un gouvernement ne fait pas ça pour rester au statu quo, y fait ça pour des économies. Si y fait ça pour des économies, y va juste avoir un poste de pompiers pour la grande municipalité. Si y garde les trois postes de pompiers, bien fais-le pas ton regroupement» (LM8).

Compte tenu du fait que dans la très grande majorité des cas, le projet de communauté rurale implique plusieurs localités, le discours portant sur les services fait surtout référence à l'aspect du regroupement, plutôt qu'à la question de la municipalisation. Mais l'accent mis sur le regroupement plutôt que sur la municipalisation montre qu'on n'associe pas directement le statut municipal à l'obtention de meilleurs services, seulement à leur gestion. S'il est donc clair qu'on associe la municipalité à son rôle de gestionnaire de ces services locaux, pour les résidents des DSL, ces services peuvent tout autant être gérés et procurés par d'autres instances, notamment en raison du fait que leurs services sont très limités. La question centrale est donc à savoir qu'elle instance est plus à même de gérer les aspects financiers liés à ces services et si la municipalisation augmenterait leurs coûts.

\section{La gestion et le partage des coûts}

La crainte de voir les impôts fonciers augmenter est sans contredit l'enjeu le plus discuté, largement partagé et relayé dans les médias, malgré le fait que pour les projets étudiés, il n'y avait en réalité pas ou peu d'augmentation projetée dans les projets proposés. Les taxes foncières sont généralement très impopulaires et considérées arbitraires (Sheffrin 2013; Sancton 2015), il n'est donc pas surprenant que tout changement anticipé à ce niveau favorise une attitude défensive.

Au Nouveau-Brunswick, l'impôt foncier est perçu par la province, mais la part municipale est décidée par le conseil municipal chaque année. Dans les DSL, c'est la province qui décide du taux et celui-ci demeure souvent inchangé pendant de nombreuses années. Comme mentionné plus haut, l'impôt des DSL ne couvre pas les coûts de leurs services (Bourgeois et Strain, 2009), mais cela n'est pas connu par les résidents, qui pour la plupart pensent payer leur part d'impôts. De plus, les résidents des DSL utilisent les services dans les municipalités avoisinantes (installations sportives, récréatives, etc.). II existe donc depuis longtemps des tensions entre les municipalités et les DSL autour du partage des coûts des services. Cet aspect du débat transparaît dans certains entretiens, comme le traduit cette remarque: "it's always been their [les municipalités] message that we [les DSL] don't pay enough» (KE4).

Cela dit, il est clair pour tous que les impôts, peu importe les taux, servent à payer les services essentiels, mais les opposants craignent que les élus locaux ne soient pas en mesure de gérer équitablement et convenablement le budget d'une éventuelle communauté rurale. On redoute l'endettement associé à des «éléphants blancs», les coûts associés à l'administration de ce palier local, ainsi que l'iniquité territoriale dans l'attribution des fonds. Dans le cas de regroupements, on ne veut pas que les impôts issus d'une localité servent à payer les services d'autres localités et cela, même si les projets de communauté rurale prévoient un fonctionnement avec des taux de taxation distincts dans chaque localité. «On sait qu'il faut qu'on paye la taxe, mais on [ne] veut pas payer la taxe pour les autres. On veut payer la taxe pour nos services, tu sais » (LM5). Ce type de crainte a d'ailleurs été relevé dans le cas de fusions de municipalités, notamment en Ontario (Kushner \& Siegel, 2003).

C'est aussi le côté arbitraire de l'impôt foncier qui le rend aussi impopulaire. Plusieurs ne pensent pas que les relevés d'impôts fonciers reflètent la valeur de leur propriété, surtout en région rurale, comme l'illustre cet extrait tiré d'une entrevue avec un résident d'une région éloignée et fortement opposé au projet proposé:
Monter les taxes? Moi j'achète [une telle maison], je [ne] veux pas payer des taxes de fou parce que ma maison est grande. Je [ne] la vendrai pas le prix qu'ils vont dire sur les taxes [compte d'impôts fonciers) (LM7)

Dans le camp des promoteurs des projets de communauté rurale, la municipalisation signifie cependant un pouvoir pour intervenir directement sur la gestion des fonds. Ce type d'argument est largement partagé: "Donc je trouvais malheureux qu'on est tous des payeurs de taxes pis on a aucune décision à prendre sur la destinée justement de nos taxes » (LM11). On espère ainsi organiser de manière plus avantageuse la prestation de services que sous la tutelle de la province. L'organisation sous forme de DSL est en effet reconnue pour donner peu de pouvoir. "My ex-husband used to call it [comite de DSL] the committee that does nothing because you couldn't, there was very little you could do » $(\mathrm{H} 3)$. Les répondants cherchent par la municipalisation en communauté rurale à acquérir un statut qui leur donnerait plus de pouvoir et de légitimité dans les décisions relatives à l'utilisation des impôts fonciers et autres revenus. Le gouvernement local est ainsi perçu comme un moyen de prendre en compte l'expression des citoyens dans la gestion des services, mais également au sein d'instances régionales et provinciales, comme l'exemplifie cet extrait: «I truly believe it's much better that we have a say in our taxes of how it's spent, we have a say in many committees provincial-wide the different service commissions and things like that, we have a seat on it which we never did before » (H6). Au-delà de la question des services et de leurs coûts, ces acteurs font ici référence au renforcement des capacités afin de gérer et promouvoir le développement local, mais surtout à l'expression politique de la communauté, ce qui constitue la deuxième grande catégorie de rôle des gouvernements locaux.

\section{La municipalité comme institution politique}

Il faut d'abord souligner que même sous l'angle de la représentation politique, il s'agit principalement pour plusieurs d'une institution plus démocratique pour gérer les fonds publics qui servent à financer les services. Ces deux citations résument bien cet état d'esprit:

Basically, municipality is put in place to provide needed community services and to permit people to have a say through the voting process, use democratic process to have a say in the decisions that have been made (KE1).

La municipalité est là pour représenter la volonté des gens de la municipalité pis pour amener des idées à la table pour faire progresser la municipalité avec le support des gens naturellement pis avec la basse de taxes, la basse d'argent qu'on a pour travailler avec (HM4).

Mais au-delà de la question des services, les opposants comme les défenseurs des projets de municipalisation reconnaissent qu'un conseil élu donne davantage de pouvoir que toute autre forme de représentation. C'est-à-dire qu'à I'heure actuelle, les résidents des DSL peuvent choisir une personne pour les représenter auprès du ministère de l'Environnement et des Gouvernements locaux et certains de ces représentants peuvent siéger à la Commission de services régionaux. Cette personne, souvent appelée président de DSL, est généralement choisie dans une assemblée à main levée. Plusieurs participants soulèvent donc le manque de légitimité des représentants de DSL. Dans les instances régionales, comme les commissions d'aménagement, ils ne sont habituellement pas traités au même titre qu'un maire: 
We don't count because we're not elected. [...] We're nominated and we're elected by the people that attend that meeting. [...] And so, we're treated, disdain is a little harder a word but... We're not taken, we're not treated with the same level of respect as what a village or a town mayor would be (H1).

La représentation par des élus n'est toutefois pas toujours considérée une vertu dans les DSL. On associe d'abord cette idée à de la bureaucratie, à une multiplication des étapes et des paliers inutile, ainsi qu'à une perte de pouvoir local.

\section{La représentation élective}

Plusieurs opposants au projet de municipalisation proposé pour leur localité ne sentent pas le besoin d'être représentés par des élus. Ceci compliquerait même la vie communautaire:

Bien un DSL c'est pas compliqué [...], certain, faut que t'as un comité de mis en place. Faut que ça soit structuré pis bien fait mais comme je t'ai expliqué tantôt la vision qu'on a de la chose nous autres si le gouvernement veut nous donner de l'aide y nous le donne mais on investit dans notre propre initiative. (LM8).

Des acteurs se questionnent sur leur capacité à négocier avec ce nouvel échelon, advenant l'émergence d'un palier municipale, au lieu de travailler directement avec le provincial:

Tu sais, les comités finissent par aller chercher les appuis où y'ont besoin directement à, la province. [...] Tu sais sans passer par une mairie qui le ferait pour eux. [...] Oui c'est ça. C'est ça qui arrive, mais c'est plus laborieux. [...] C'est plus laborieux, mais ça permet plus de possibles que de passer par la mairie (LM3).

Mais les DSL eux autres avons aucune forme de gouvernement. On est représenté par un gars du gouvernement qui décide pour nous autres, on fait des demandes pis y'accepte nos demandes. Ça vraiment bien fonctionné ça. Ça fonctionnait vraiment bien, tu sais j'veux dire (LM5).

Dans la même veine, un aspect bureaucratique est largement associé au gouvernement municipal:

Moi j'dis [...] le plus grand qu'une compagnie devient, le plus que c'est difficile à prendre soin et le plus longtemps que ça prend avant de passer à travers la hiérarchie pour avoir tes réponses ou avoir ton travail fait (HM5).

Dans les cas étudiés, des comités de bénévoles non élus craignent en fait de devoir se subordonner à des instances élues en cas de municipalisation: "les gens se sentent dépossédés de leur pouvoir décisionnel à cause que ça fonctionne en p'tits comités pis on est capable des fois de gérer en p'tits comités [sans la municipalité] » (LM3).

Pourtant la collaboration entre la municipalité (élus et gestionnaires) et les acteurs du milieu (associations locales et comités) est sou- vent au cœur du projet du territoire (Landel \& Pecqueur 2014). Les porteurs des projets de communauté rurale avancent d'ailleurs pour leur part qu'une municipalité sera davantage en mesure de soutenir le travail des acteurs communautaires et privés dans leurs efforts de développement. Mais il peut exister des contradictions et des tensions entre l'action collective relevant des acteurs du milieu, municipalité incluse, et l'action publique portée par la municipalité (Belley 2016) et il semble que plusieurs opposants impliqués dans le développement de leur localité craignent justement cette trajectoire.

En fait, partisans comme opposants aux projets de municipalisation reconnaissent la légitimité du modèle de démocratie représentative. Mais certains préfèrent tout de même le maintien du statu quo afin de ne pas se faire "gouverner». Car, dans les territoires non municipalisés, plusieurs s'inquiètent de ce que pourrait imposer un gouvernement local. Par exemple des habitants des DSL ne veulent pas se faire prescrire des «règlements de ville » jugés trop contraignants: "On perdrait notre liberté de vivre en campagne [...] Ça aurait été deux îles une ville. [...] On aurait été sous les lois municipales» (LM5). Malgré une autonomie effective plutôt faible, des participants craignent de perdre le contrôle sur leur destinée avec un gouvernement beaucoup plus proche d'eux.

Cette crainte est encore plus explicite lorsque la question de la composition d'un éventuel conseil municipal est abordée. Dans le cas des regroupements, plusieurs s'inquiètent du fait que les décisions au sujet d'un projet situé dans leur communauté soient prises par une majorité de conseillers qui résident dans d'autres localités regroupées au sein d'une communauté rurale.

La nouvelle grande municipalité, bien nous autres on n'a pu de pouvoir décisionnel. T'as une personne là pis eux autres sont majoritaires au conseil de la grande municipalité. [...] Fait que mettons qu'une compagnie comme ça [d'exploitation de tourbière] va voir la grande municipalité pis y dit moi j'suis prêt à investir bien nous autres on n'a pu de contrôle dessus (LM8).

Les extraits d'entretiens faisant référence à cette peur de se faire gouverner par les communautés voisines sont très fréquents. Cela s'explique en partie par le fait que pour respecter des seuils de population et d'assiette fiscale minimums fixés par le gouvernement provincial, les projets de communautés rurales regroupent souvent plusieurs localités sur de larges territoires et que dans les projets proposés, il n'y a pas plus qu'un ou deux élus par localité au conseil.

Les discussions intenses autour de la représentation de chaque localité au sein des futurs conseils, frontières des quartiers et poids électoral, reflètent cette crainte de ne pas être reconnus comme des territoires ayant des besoins distincts des autres. Cependant plusieurs admettent que certains projets ou services seraient mieux portés par un collectif plus large, mais ils aimeraient alors qu'au sein de ce regroupement, on leur garantit une certaine autonomie (ex: droit de veto), comme l'explique une participante: "Something where we might have the rural community as an overarching body but under it we still have our own local decision-making » (KE5). Pour plusieurs, le fonctionnement actuel basé sur des ententes de services entre collectivités, au cas par cas, est alors adéquat.

Chez les acteurs en faveur des projets de municipalisation, le discours est tout autre et plus proche des rôles typiquement attribués à ce palier de gouvernement aujourd'hui. On y voit un gouvernement de proximité, une institution garante du développement de la collectivité et capable d'exprimer et représenter ses intérêts: 
Just having a local government, a place where you can go, a council that cares about the residents and the needs of the community, I think that's always very very beneficial $(\mathrm{H} 4)$.

I have better access to my mayor and council whereas before mayor and council was whoever the minister of local government was and at that time we incorporated with Bruce Fitch but you know, I couldn't just pick up the phone and call Bruce but now I can pick up the phone and call the mayor or I can text him so I have better access to those in leadership which makes me feel more comfortable so if there is an issue, I have a direct line now and there's an office I can go to if I want to complain or ask a question whereas before, I didn't know how to do that. (H10)

Ah lorsque j'ai vu les bienfaits à la table d'un regroupement possible lorsqu'on parle par exemple qu'on a une destinée, le fonds de taxes sur l'essence, les économies d'échelle avec la fusion des municipalités, les services qu'on pouvait jumeler et tout ça, l'embauche d'un agent de développement touristique et économique qui était aussi proposé dans le projet (LM11).

Cette dernière citation résume en effet bien les avantages promus par les partisans des projets de création de communauté rurale.

\section{La consistance du collectif}

Pour arriver à concevoir les avantages précédemment cités, il faut avoir une vision étendue de la communauté. En effet, la question de la consistance même de la communauté, de sa définition, de ses frontières, relève du politique.

Au-delà de la politique associée au gouvernement, qu'il soit local ou autre, et à la représentation par des élus, nous pouvons dégager de notre analyse de contenu, cette idée que les communautés locales sont capables d'agir sur elle-même. Bien que certaines remarques au sujet de l'opposition à de nouveaux règlements qui viendraient avec l'installation d'un gouvernement local donnent une impression de libertarisme, l'aspect collectif est très présent, mais s'exprime à une autre échelle.

La définition de projets collectifs implique que des acteurs «partagent une identité commune en relation à un territoire, une conscience territoriale qui les amène à réaliser des projets en association, » (Klein, 2006: 156). Plusieurs exemples de reformes des administrations territoriales mettent bien en évidence les liens existants entre l'appartenance au territoire et à l'administration locale, cette appartenance est notamment utilisée pour s'opposer à des fusions (Hall \& Stern, 2009; Mévellec, 2008, Tomàs \& Collin, 2005, Bherer \& Lemieux, 2002). Mais la relation identitaire à un territoire et l'élaboration de projets collectifs vont bien au-delà du lien existant entre l'acteur municipal et les autres acteurs du territoire.

En ce qui concerne les enjeux associés aux projets de création de communauté rurale, tant chez les opposants que chez les partisans, les discours sur l'appartenance sont souvent liés au statut administratif (DSL ou municipalisé) actuel, convoité ou rejeté. Dans le camp des opposants, les discours relatifs aux enjeux d'appartenance révèlent non seulement une crainte du changement d'échelle du collectif qu'entrainerait le regroupement, mais aussi une autre forme de revendication identitaire: la ville (le territoire ordonné des élites, la bureaucratie) contre la campagne (le DSL, un espace de communauté), et ce même si dans les faits la «ville» est plutôt de la taille d'un village dans d'autres juridictions. Le N.-B. ne fait par ailleurs pas exception au classique «effet de clocher » déjà mis relief dans l'ana- lyse de cas de fusions (Tomàs \& Collin 2005; Quesnel et al., 2005; Bherer \& Lemieux, 2002).

La construction de l'appartenance à une communauté semble être assez peu associée à l'existence d'un gouvernement local. Mais les partisans de la municipalisation estiment pour leur part que la création d'une communauté rurale sera bénéfique au renforcement et à la construction d'un sentiment d'appartenance à une collectivité élargie. Pour une participante, être municipalisé permet «de développer un sens d'appartenance [...] c'est leur édifice municipal, c'est là qu'ils se regroupent, c'est là qu'est le cœur de la municipalité [...] le sentiment d'appartenance c'est quelque chose qui est vraiment important » (LM10). L'expérience récente de Hanwell semble aussi aller dans le sens d'un rôle important de la municipalité dans ce processus de construction identitaire, comme l'explique cette participante:

So one of the biggest challenges that we have to overcome is because we are a newly incorporated community is to strengthen our identity and to pull our neighbours together. [...] and we're doing a great job. Like since we've been incorporated just in the last 2 years, we are seeing people come out, like the attendance to our events has gone through the roof when we host a tree-lighting ceremony and open house (H7).

Dans le cas du projet du Haut-Madwaska, le processus de création de la communauté rurale a servi de base à une définition de projets qui font référence à des éléments identitaires communs.

On a utilisé tous les thèmes qui faisaient en sorte que ça venait chercher le cœur pis ça venait chercher la tête, écoute faut. Donc pour ça moi, le comité et moi, [...], on jubile devant cette dynamique-là qu'on a réussi à mobiliser autant de population à un projet commun et c'est ce qui fait en sorte que ça va définitivement aider le nouveau conseil municipal à travailler et à amener ces gens-là à travailler ensemble (HM6).

En résumé, souvent, pour les promoteurs de la communauté rurale, la construction d'un sentiment d'appartenance à une communauté est un aspect important du projet, mais les opposants n'envisagent pas ces changements positivement. Ces discours soulignent l'influence de la réflexion sur la définition de la communauté dans le positionnement des acteurs par rapport au statut administratif (DSL ou municipalité).

\section{CONCLUSION}

L'analyse des entrevues conduites auprès de partisans et d'opposants aux projets de création d'une communauté rurale nous a permis de mieux comprendre l'environnement discursif au sujet du rôle des municipalités qui se développe autour des projets visant essentiellement la municipalisation de localité sans gouvernement local, passant la plupart du temps par un regroupement de localités (DSL et petites municipalités déjà existantes).

Comme ailleurs, l'efficience et la performance de la gestion des services et enjeux locaux sont centrales dans les projets de réformes de la gouvernance locale (Landel \& Pecqueur, 2014; Lesage \& Garcea, 2005; Vojnovic \& Poel, 2000). Même en France où la dimension politique prend plus d'importance qu'en Amérique (Mévellec et al., 
2017), les projets d'intercommunalité visent à augmenter l'efficacité des services, sans que ne soit posée la question de la citoyenneté (Landel \& Pecqueur, 2014).

Les résultats de cette étude vont dans le même sens que les travaux de Chiasson et al., (2014) et le rapport Finn (2008) : les municipalités sont surtout vues comme des pourvoyeuses de services, et ce par les tenants comme les opposants à la municipalisation. Les premiers adhèrent au discours d'une rationalisation des coûts possibles à travers des regroupements ainsi qu'au principe de subsidiarité assumant qu'un pouvoir de gestion local sera plus à même de répondre aux besoins de la collectivité. Les seconds remettent en question la possibilité d'optimiser les services sans qu'il ait d'augmentation des taxes foncières. Pour plusieurs opposants, la gestion centralisée du peu de services qu'ils ont n'est pas un problème. Dans un contexte de compression budgétaire où les collectivités se retrouvent en compétition les unes avec les autres, les habitants des plus petites communautés craignent que la création de communautés rurales les défavorise. En réponse à ce même discours, les porteurs des projets de communautés rurales voient plutôt ces démarches comme une planche de salut pour négocier avec la province et gérer eux-mêmes les services sur leur territoire. Dans les deux cas, sous-jacents à la simple question de la gestion des services, émerge rapidement le rôle politique d'un gouvernement local.

La composition d'une institution démocratique fondée sur une représentation élective permettrait, pour les promoteurs de la municipalisation, de faire des choix au sein de son territoire et de défendre ses choix auprès des autres instances gouvernementales. Mais pour les opposants, cette représentation est souvent appréhendée comme une éventuelle perte de pouvoir local. Ainsi, plusieurs répondants ne semblent pas vouloir déléguer à un conseil municipal, qui plus est fondé sur la fusion de plusieurs communautés, la responsabilité du collectif.

Cette dimension politique fait ainsi émerger des enjeux identitaires associés à la définition de la communauté qui ne sont pas forcément tributaires de l'encadrement par une institution municipale. Comme dans le cas de fusion ailleurs, la résistance peut être liée à une forte identité et un fort sentiment d'appartenance local (Hall \& Stern, 2009) - dans ce cas archi-local - alors l'importance de se doter d'un gouvernement local devient d'autant plus relative. Par ailleurs, cinquante ans de fonctionnement avec un modèle d'organisation du territoire centralisé, et perçu comme distant, a aussi entraîné la construction de référents particuliers: l'absence de gouvernement local est devenue normale, car les services locaux, puisque peu nombreux, peuvent être gérés et procurés par d'autres instances.

Finalement, il nous apparaît que pour que l'adhésion aux projets de municipalisation augmente, il faudrait éventuellement que des exemples de démocratie délibérative ancrés dans les localités et le fonctionnement des municipalités soient davantage nombreux, connus et surtout valorisés par tous les acteurs, incluant la province.

\section{RÉFÉRENCES BIBLIOGRAPHIQUES}

Andrew, C. \& M. Goldsmith (1998). From Local Government to Local Governance and beyond? International Political Science Review 19(2): 101-117.

Antoft, K. \& J. Novack.1998. Grassroots Democracy, Local Government in the Maritimes, Halifax: Dalhousie University.

Belley, S. (2016). Plus autonomes, les municipalités québécoises seront-elles en mesure de relever les nombreux défis de la gouvernance de proximité? Revue vie économique 8: 1-8.

Bherer, L. \& V. Lemieux (2002). La référence aux valeurs dans le débat sur la réorganisation municipale au Québec. Revue canadienne des sciences régionales / Canadian Journal of Regional Science 25 (3): 447-472.

Bourgeois, D. (2005). Municipal Reforms in New Brunswick: To decentralize or not to Decentralize?, in E. Lesage \& J. Garcea (ed.), Municipal Reforms in Canada: Municipal Governance for the $21^{1 \mathrm{t}}$ Century, Oxford: University Press/Don Mills: 242-68.

Bourgeois, D. (2009). La prestation des services bilingues au Canada, in M. Martel \& M. Pâquet (dir.), Légiférer en matière linguistique: Pour qui? Pourquoi? Comment? Québec: Presses de I'Université Laval: 267-294.

Bourgeois, D. \& F. Strain (2009). New Brunswick, In A. Sancton \& R. Young (ed.) Foundations of Governance:Municipal Government in Canada's Provinces. Toronto: University of Toronto Press: 186-222.

Chiasson, G., Y. Fournis \& A. Mévellec (2014). Fermer la parenthèse régionale: retour au municipal! Économie et Solidarités 44 (1-2): 64-83.

Cleave, E. \& G. Arku (2015). Place branding and economic development at the local level in Ontario, Canada. GeoJournal 80 (3): 323-338.

Courlet, C. \& B. Pecqueur (2013). L'économie territoriale. Grenoble: Presses universitaires de Grenoble.

Cyr, G. (2001). La réforme municipale de 1967 au Nouveau-Brunswick, in L'ère Louis J. Robichaud 1960-1970, actes du colloque, 8-9 septembre 1999. Moncton: Institut canadien de recherche sur le développement régional, 143-167.

Douglas, D. (2005). The restructuring of local government in rural regions: A rural development perspective. Journal of Rural Studies, 21: $231-246$.

Finn, J.-G. (2008). Bâtir des gouvernements locaux et des régions viables: plan d'action pour l'avenir de la gouvernance locale au Nouveau-Brunswick, Fredericton: Ministère des gouvernements locaux.

Foucher, P. (2012). Autonomie des communautés francophones minoritaires du Canada: le point de vue du droit. Minorités linguistiques et société 1: 90-114.

Garcea, J. \& Edward C. L. Jr. (ed.) (2005). Municipal Reform in Canada: Reconfiguration, Re-Empowerment, and Rebalancing. Toronto: Oxford University Press.

Gauthier, B. (2000). La structure de la preuve in B. Gauthier (ed.), La structure de la preuve. Recherche sociale. De la problématique à la collecte des données. Québec: Presse de I'Université du Québec, 127-158

GNB (2007). Le Groupe de travail sur l'autosuffisance publie son troisième rapport (07/03/09): https://www.gnb.ca/cnb/newsf/sst/ 2007f0312tf.htm

Guillemot, J. (2017). La gouvernance des territoires: obstacles et opportunités en Acadie. Port Acadie 28: 59-78

Hall, P. V. \& P. Stern (2009). Reluctant rural regionalists. Journal of Rural Studies 25: 67-76.

Klein, J.-L. (2006). De l'initiative locale au développement territorial: une perspective synthétique, in Simard M., D. Lafontaine, S. Savard, M. Tremblay \& P.-A. Tremblay (ed.), Inégalités, démocratie et développement - Des enjeux pour la gouvernance des territoires locaux et régionaux. Rimouski : GRIDEQ.

Kushner, J. \& D. Siegel (2005). Are Services Delivered More Efficiently After Municipal Amalgamations?, Canadian Public Administration / Administration Publique du Canada 48 (2): 251-67.

Kushner, J. \& D. Siegel (2003). Citizens Attitudes Toward Municipal Amalgamation in Three Ontario Municipalities. Canadian Journal of Regional Science 26 (1): 49-59. 
Landel. P. \& B., Pecqueur (2014). Décentralisation à la française et innovation sociale: un compromis impossible. Économie et Solidarités 44 (1-2): 69-182.

Landry, M. (2007). Le nouveau projet de communautés rurales au Nouveau-Brunswick: une occasion d'acquisition de pouvoirs et d'autonomie pour les Acadiens. Francophonies d'Amérique 23-24: 15-29.

Lemire, F., D. Juteau, S. Arcand \& S. Bilge (2005). Le débat sur la réforme municipale à Montréal. La place de la variable linguistique. Recherches sociographiques 46 (1): 67-96.

Lesage, E. C. \& J. Garcea (2005). Reflections on Municipal Reform: Reconfiguration or Reinvention?, in J. Garcea \& E. C. Lesage (ed.), Municipal Reform in Canada. Toronto: Oxford University Press, 289341.

McAllister, M. L. (2004), Governing Ourselves? The Politics of Canadian Communities, Vancouver: UBC Press.

McKay, R. B. (2004). Reforming municipal services after amalgamation: The challenge of efficiency. The International Journal of Public Sector Management 17 (1): 24.

Martin, G. R. (2007). Municipal Reform in New Brunswick: Minor Tinkering in Light of Major Problems. Journal of Canadian Studies / Revues d'études canadiennes, 41(1): 75-100.

Mévellec, A. (2008). La construction politique des agglomérations au Québec et en France. Québec: Presses de l'Université Laval.

Mévellec, A. \& V. B. (2003). Fusions municipales au Québec, coopération intercommunale en France: deux approches convergentes? Pouvoirs Locaux, 58(3): 116-122.

Mévellec, A., G. Chiasson \& Y. Fournis (2017). De «créatures du gouvernement » à «gouvernements de proximité » : la trajectoire sinueuse des municipalités québécoises ». Revue française d'administration publique 2 (162): 339-352

Neuman, W. L. (2010). Social Research Methods. Qualitative and Quantitative Approaches ( $7^{\text {th }}$ edition). Londres: Pearson.

Quesnel, L., Bherer, L \& Sénéchal, M.-R. (2005). Fusion municipale et territoire de proximité, in L. Bherer, J.-P. Collin, É. Kerrouche and J. Palard (ed.), Jeux d'échelle et transformation de l'État: le gouvernement des territoires au Québec et en France. Québec: Les presses de l'université Laval, 427-452.

Rosenfeld, R. A. \& Reese, L. A. (2003). The Anatomy of an Amalgamation: The Case of Ottawa. State and Local Government Review 35 (1): 57-69

Sancton, A. (2000). Amalgations, service realignements and property taxes: Did the Harris government have a plan for Ontario's municipalities? Revue canadienne des sciences régionales 23: 135-156.

Sancton, A. (2015). Canadian Local Government. An Urban Perspective (second edition). Don Mills (On): Oxford University Press.

Sancton, A. \& R. Young (ed.) (2009). Foundations of Governance: Municipal Government in Canada's Provinces. Toronto: University of Toronto Press.

Savoie-Zajc, L. (2008). L'entrevue semi-dirigée, in B. Gauthier (ed.), Recherche sociale, De la problématique à la collecte des données (5e édition). Québec: Presses de l'Université Laval, 337-360

Sheffrin, S.M. (2013). Tax Fairness and Folk Justice. New York: Cambridge University Press.

Spicer, Z. (2012). Post-Amalgamation Politics: How Does Consolidation Impact Community Decision-Making?, Canadian Journal of Urban Research 21 (2): 90-111.

Statistique Canada (2016). GéoSuite, Recensement de 2016, produit no 92-150-X au catalogue de Statistique Canada.
Statistique Canada (2006). Recensement de 2006. Ottawa: Gouvernement du Canada

Tomàs, M. \& J.-P. Collin (2005). Constance et mutation: le discours des acteurs municipaux devant le mouvement «défusionniste» au Québec. Canadian Journal of Regional Science/Revue canadienne des sciences régionales XXVIII (1):139-160.

Tindal, R. C., S. Nobes Tindal, K. Stewart \& P. J. Smith (2013). Local Government in Canada (8e édition). Scarborough (ON): Nelson.

Vojnovic, I. \& P., Dale (2000). La restructuration provinciale-municipale au Canada: une évaluation des attentes et des résultats. Canadian Journal of Regional Science/Revue canadienne des sciences régionales XXIII (1): 7-12.

Young, R. (2001). Le programme Chances égales pour tous: une vue d'ensemble, in L'ère Louis J. Robichaud 1960-1970, actes du colloque, 8-9 septembre 1999, Moncton, Institut canadien de recherche sur le développement régional, 23-37.

Young, R. (2013). La gouvernance multiniveau et les politiques publiques au sein des municipalités du Canada: reddition de comptes et efficacité. Télescope: Revue d'analyse comparée en administration publique 19 (1): 25-42. 